\title{
Construction of continuous abstractions for discrete-time time-delay systems
}

\author{
Mobin Shahamat, Javad Askari, Abdalla Swikir, Navid Noroozi and Majid Zamani
}

\begin{abstract}
In this paper we construct continuous abstraction for discrete-time time-delay systems via the notion of so-called Razumikhin simulation functions. We show that the existence of such a function guarantees that the mismatch between the output trajectory of the concrete system and that of its abstraction lies within an appropriate bound. By transforming a system with time delay into an interconnected system without time delay, we show that the Razumikhin method is a small-gain type approach for time-delay systems and enables us to effectively manage computational complexity of constructing abstractions. We further extend our approach to compositional construction of large-scale systems containing interconnection and/or local time delays. For linear systems, we provide an algorithmic procedure for compositional construction of abstractions, which is expressed in terms of linear matrix inequalities.
\end{abstract}

\section{INTRODUCTION}

Time delays arise in natural and engineering systems, e.g. biology, chemistry, economics, electronics, and mechanics due to a wide variety of effects [1]. For instance, they may be because of the propagation of physical quantities over large distances, or modeling complex physical effects such as viscoelasticity, finite reaction rates, and polymer crystallization. In addition, actuators and sensors connected to plants and the implementation of a controller over a communication network with a limited bandwidth usually introduce time delays. In particular, control of discrete-time systems with time delays have received considerable attention in a large number of applications including networked control systems, neural networks, and multi-agent systems [2].

Recently there has been great deal of attention to automated synthesis of provably correct controllers by merging ideas from computer science and control theory In particular, formal synthesis techniques can effectively reduce the costs of incorrect configuration and address safety concerns and other related complexities raised by emerging smart applications. Such techniques usually require a symbolic model of

M. Shahamat and J. Askari are with the Department of Electrical and Computer Engineering, Isfahan University of Technology, 8415683111 Isfahan, Iran, m. shahamateec.iut.ac.ir, j-askarieiut.ac.ir.

A. Swikir is with the Department of Electrical and Computer Engineering, Technical University of Munich, Germany, abdalla.swikiretum.de.

N. Noroozi is with the Institute of Informatics, LMU Munich, Germany, navid.noroozi@lmu.de. His work is supported by the DFG through the grant WI 1458/16-1.

M. Zamani is with the Computer Science Department, University of Colorado Boulder, CO 80309, USA. M. Zamani is also with the Institute of Informatics, LMU Munich, Germany, majid.zamani@colorado.edu. His work is supported in part by the DFG through the grant ZA 873/4-1 and the H2020 ERC Starting Grant AutoCPS (grant agreement No. 804639). a given concrete system in the form of a discrete abstraction. Here a discrete abstraction is a model over a finite state set such that there exists a quantifiable relation between the dynamics of the concrete system and its abstraction. However, high computational complexity is their main issue. Thus, in practice they can only be applied to control systems with small state space dimension. An efficient way to reduce this computational complexity is to introduce a pre-processing step by constructing a so-called continuous abstractions. In this way, a continuous-space system, but possibly with a lower dimension, is obtained for the concrete system.

To compute abstractions, the notion of approximate simulation relations and their variants have been particularly leveraged; see for example [3], [4]. This notion relaxes its exact counterpart [5], [6] by allowing for the mismatch between the output trajectory of the concrete and that of the abstract systems to be below an acceptable bound instead of being strictly zero. For systems without delay approximate simulation relations can be quantitatively characterized by a Lyapunov-like function called a simulation function [4], [7]. However, to the best of our knowledge, this technique has not been adapted to systems with time delay. Note that the works in [8]-[10] have developed monolithic construction of (alternatingly) approximately bisimilar discrete abstractions for time-delay systems in continuous-time domain by leveraging notions of incremental input-to-state stability. In this paper we address the construction of approximately similar continuous abstractions of time-delay systems in discretetime domain both in monolithic and compositional ways.

Here, we extend the result in [7] to discrete-time systems with time delay in two directions. Motivated by Lyapunov methods for stability analysis of time delay systems [11], we introduce a concept of Razumikhin simulation functions to monolithically construct approximations of a single timedelay system. The main idea, motivated by the results in [12], is to transform a single system with time delay into an interconnected system without time delay by defining each delayed state as a new subsystem. This enables us to exploit a compositionality approach to construct abstractions of time-delay systems via standard small-gain arguments for interconnected systems as in [13], [14]. In that way, for each subsystem of the interconnected system we construct a local abstraction. Then we aggregate local abstractions to generate an abstraction of the overall system if the coupling between subsystems are small enough, which is quantitatively expressed by a small-gain condition. The whole procedure boils down to the notion of Razumikhin simula- 
tion functions, which shows that the Razumikhin simulation method is an exact application of small-gain theory [15] to systems with time delay. Then we further extend our result to interconnected systems, where we consider both local and interconnection delays. For linear systems, we provide algorithmic procedures explicitly constructing an abstraction for the linear concrete system. We verify the effectiveness of our proposed results via an illustrative example. All proofs are omitted due to space constraints

\section{PRELIMINARIES}

\section{A. Notation}

Let $\mathbb{R}_{\geq 0}\left(\mathbb{R}_{>0}\right)$ and $\mathbb{N}_{0}(\mathbb{N})$ denote the non-negative (positive) real numbers and the non-negative (positive) integers, respectively. The vector space of real column vectors of length $n$ is denoted by $\mathbb{R}^{n}$. We denote the closed, open, and half-open intervals in $\mathbb{R}$ by $[a, b],(a, b),[a, b)$, and $(a, b]$, respectively. For $a, b \in \mathbb{N}$ and $a \leq b$, we use $[a ; b],(a ; b)$, $[a ; b)$, and $(a ; b]$ to denote the corresponding intervals in $\mathbb{N}$. Given $N \in \mathbb{N}$, vectors $v_{i} \in \mathbb{R}^{n_{i}}, n_{i} \in \mathbb{N}$, and $i \in[1 ; N]$, we use $v=\left[v_{1} ; \ldots ; v_{N}\right]$ to denote the vector in $\mathbb{R}^{n}$ with $n=\sum_{i} n_{i}$ consisting of the concatenation of vectors $v_{i}$. Furthermore, the $i$ th component of $v \in \mathbb{R}^{n}$ is denoted by $v_{i}$. For any $v \in \mathbb{R}^{n}, v^{\top}$ denotes its transpose. The Euclidean norm of a vector $v \in \mathbb{R}^{n}$ is denoted by $|v|$; for notational convenience we use the same symbol for the matrix norm. Given a function $\nu: \mathbb{N}_{0} \rightarrow \mathbb{R}^{n}$, the supremum of $\nu$ is denoted by $|\nu|_{\infty}$; we recall that $|\nu|_{\infty}:=\sup _{k \in \mathbb{N}_{0}}|\nu(k)|$. Given a real symmetric matrix $N \in \mathbb{R}^{n \times n}, N \succ 0(N \succeq 0)$ denotes the property that $x^{\top} N x>0\left(x^{\top} N x \geq 0\right)$ for all $x \neq 0$.

Let id denotes the identity function. We will consider $\mathcal{K}, \mathcal{K}_{\infty}$, and $\mathcal{K} \mathcal{L}$ comparison functions, see [16, Chapter 4.4] for definitions. For functions $\alpha, \gamma: \mathbb{R}_{\geq 0} \rightarrow \mathbb{R}_{\geq 0}$ we write $\alpha<\gamma$ if $\alpha(s)<\gamma(s)$ for all $s>0$.

\section{B. A hierarchical control approach}

Consider the control system given by

$$
\Sigma:\left\{\begin{aligned}
\mathbf{x}(k+1) & =f(\mathbf{x}(k), \mathbf{u}(k)), \\
\mathbf{y}(k) & =g(\mathbf{x}(k)),
\end{aligned}\right.
$$

where $\mathbf{x}: \mathbb{N}_{0} \rightarrow \mathbb{R}^{n}, \mathbf{y}: \mathbb{N}_{0} \rightarrow \mathbb{R}^{q}$, and $\mathbf{u}: \mathbb{N}_{0} \rightarrow \mathbb{R}^{m}$ are the state signal, output signal, and input signal, respectively, and the dynamics $f: \mathbb{R}^{n} \times \mathbb{R}^{m} \rightarrow \mathbb{R}^{n}$. Moreover, let $\mathcal{U}$ denote the set of all bounded input functions $\mathbf{u}: \mathbb{N}_{0} \rightarrow \mathbb{R}^{m}$. Let $\mathbf{x}(k, x, \mathbf{u})$ denote a point reached at time $k \in \mathbb{N}_{0}$ from initial state $x=\mathbf{x}(0) \in \mathbb{R}^{n}$ under input signal $\mathbf{u} \in \mathcal{U}$. Correspondingly, define $\mathbf{y}(k, x, \mathbf{u}):=g(\mathbf{x}(k, x, \mathbf{u}))$.

System (1), which is the system we aim to control, is referred as the concrete system. The controller design is, however, based on a simpler description of the concrete system called abstract system. The abstract system is defined and described, similarly to system $\Sigma$ in (1), by

$$
\hat{\Sigma}:\left\{\begin{aligned}
\hat{\mathbf{x}}(k+1) & =\hat{f}(\hat{\mathbf{x}}(k), \hat{\mathbf{u}}(k)), \\
\hat{\mathbf{y}}(k) & =\hat{g}(\hat{\mathbf{x}}(k)),
\end{aligned}\right.
$$

where $\hat{\mathbf{x}}(k) \in \mathbb{R}^{\hat{n}}, \hat{\mathbf{y}}(k) \in \mathbb{R}^{\hat{q}}$, and $\hat{\mathbf{u}}(k) \in \mathbb{R}^{\hat{m}}$.
Concrete and abstract systems can be related to each other via a simulation relation. Simulation relations have been developed for discrete systems [5]. Approximate version of simulation relations being applicable to a large class of systems have been introduced in [4]. Lyapunov-like functions, known as simulation functions, have been introduced in [4] as a quantitative generalization of approximate simulation relations. Similarly, a simulation function of $\hat{\Sigma}$ by $\Sigma$ is a function over their state spaces explaining how a state trajectory of $\hat{\Sigma}$ can be transformed into a state trajectory of $\Sigma$ such that the distance between the associated output trajectories remains within some computable bounds. Simulation function for continuous-time systems is defined in [7]. For discrete-time systems, a simulation function is defined as follows.

Definition II.1 Consider systems $\Sigma$ and $\hat{\Sigma}$ with the same output spaces. A function $V: \mathbb{R}^{\hat{n}} \times \mathbb{R}^{n} \rightarrow \mathbb{R}_{\geq 0}$ is called a simulation function from $\hat{\Sigma}$ to $\Sigma$ if there exist $\underline{\alpha}, \alpha \in \mathcal{K}_{\infty}$ with $\alpha<$ id and $\gamma \in \mathcal{K}$ such that for every $x \in \mathbb{R}^{n}, \hat{x} \in \mathbb{R}^{\hat{n}}$, $\hat{u} \in \mathbb{R}^{\hat{m}}$ there exists $u \in \mathbb{R}^{m}$ so that the following hold

$$
\begin{gathered}
\underline{\alpha}(\mid \hat{g}(\hat{x}-g(x) \mid) \leq V(\hat{x}, x), \\
V(\hat{f}(\hat{x}, \hat{u}), f(x, u)) \leq \max \{\alpha(V(\hat{x}, x)), \gamma(|\hat{u}(k)|)\}
\end{gathered}
$$

The following theorem shows that a simulation function can be used to bound the distance between output trajectories of $\Sigma$ and $\hat{\Sigma}[7],[14]$.

Theorem II.2 Consider systems $\Sigma$ and $\hat{\Sigma}$ with the same output spaces. Let $V$ be a simulation function from $\hat{\Sigma}$ to $\Sigma$. Then there exist $\beta \in \mathcal{K} \mathcal{L}$ and $\gamma \in \mathcal{K}$ such that for all $x \in \mathbb{R}^{n}$, all $\hat{x} \in \mathbb{R}^{\hat{n}}$, all $\hat{\mathbf{u}} \in \hat{\mathcal{U}}$ there exists $\mathbf{u} \in \mathcal{U}$ so that for all $k \in \mathbb{N}_{0}$ we have

$|\hat{\mathbf{y}}(k, \hat{x}, \hat{\mathbf{u}})-\mathbf{y}(k, x, \mathbf{u})| \leq \max \left\{\beta(V(\hat{x}, x), k), \gamma\left(|\hat{\mathbf{u}}|_{\infty}\right)\right\}$.

\section{RAZUMikhin Simulation FunCtion}

In this section, a new notion of simulation function, called Razumikhin simulation function, is introduced for time delay systems. We show that the existence of such a function ensures that the mismatch between the output trajectory of the concrete systems and that of the abstraction lies within an appropriate bound.

Classic analysis of discrete-time systems with time delay is to augment the state vector with all delayed states/inputs that affect the current state, which yields a standard discretetime system of higher dimension without time delay. In that way, one could monolithically analyze the mismatch between output trajectories of the resulting augmented system obtained from the concrete system with time delay and that of the abstraction. However, such a methodology will not be efficient as not only we dramatically increase the dimension of the concrete system with the increase in the amount of time delay, but also structural connection between the abstraction obtained from such an augmented and the original concrete system will be lost.

Here we propose a computationally efficient approach by looking at a system with delay as an interconnected 
system without delay. This enables us to exploit a compositionality approach to construct abstractions for time-delay systems via small-gain theorems for interconnected systems. In particular, we show that the Razumikhin approach to construct abstractions for systems with time delay is an exact application of the small-gain theorem. The latter is in line with similar observations in the literature of stability analysis of time delay systems [12], [17].

Consider the following nonlinear time delay system

$$
\Sigma^{d}:\left\{\begin{aligned}
\mathbf{x}(k+1) & =f\left(\mathbf{x}_{[k-h ; k]}, \mathrm{u}_{[k-h ; k]}\right) \\
\mathbf{y}(k) & =g(\mathbf{x}(k))
\end{aligned}\right.
$$

where $\mathbf{x}_{[k-h ; k]}=[\mathbf{x}(k-h) ; \ldots ; \mathbf{x}(k)] \in\left(\mathbb{R}^{n}\right)^{h+1}$, $\mathbf{u}_{[k-h ; k]}=[\mathbf{u}(k-h) ; \ldots ; \mathbf{u}(k)] \in\left(\mathbb{R}^{m}\right)^{h+1}, \mathbf{x}: \mathbb{N}_{0} \rightarrow$ $\mathbb{R}^{n}, \mathbf{y}: \mathbb{N}_{0} \rightarrow \mathbb{R}^{q}$, and $\mathbf{u}: \mathbb{N}_{0} \rightarrow \mathbb{R}^{m}$ are the state signal, output signal, and input signal, respectively, and $h \in \mathbb{N}$ is the maximal delay. Similar to systems without delay, let $\mathbf{x}\left(k, \mathrm{x}_{[-h ; 0]}, \overline{\mathbf{u}}\right)$ denote a point reached at time $k \in \mathbb{N}_{0}$ from initial states $\mathbf{x}_{[-h ; 0]}=[\mathbf{x}(-h) ; \ldots ; \mathbf{x}(0)]$ under input function $\overline{\mathbf{u}}$. Correspondingly, define $\mathbf{y}\left(k, \mathrm{x}_{[-h ; 0]}, \overline{\mathbf{u}}\right):=$ $g\left(\mathbf{x}\left(k, \mathbf{x}_{[-h ; 0]}, \overline{\mathbf{u}}\right)\right)$. Moreover, let $\mathcal{U}$ denote the set of all bounded input functions $\overline{\mathbf{u}}: \mathbb{N}_{0} \rightarrow\left(\mathbb{R}^{m}\right)^{h+1}$.

Due to the possibly high dimensionality of the concrete system, a controller design will be preferably carried out based on a simpler form of the concrete system called an abstract system, denoted by $\hat{\Sigma}^{d}$, which can be also given by adopting the same notational convention, but the sign $\hat{\cdot}$ on the top of the symbols in (5).

Our aim is to introduce a quantitative relation between concrete and abstract systems characterizing the mismatch between the output trajectories of the two systems. For systems without time delay this is given by the notion of simulation function which is inspired by the concept of Lyapunov functions from the literature of control theory.

For systems with time delay the notion of RazumikhinLyapunov function has been widely used in control theory literature [11]. Therefore, with a similar methodology we introduce the notion of Razumikhin simulation functions.

Definition III.1 Consider systems $\Sigma^{d}, \hat{\Sigma}^{d}$ with same output spaces. A function $V: \mathbb{R}^{\hat{n}} \times \mathbb{R}^{n} \rightarrow \mathbb{R}_{\geq 0}$ is called a Razumikhin simulation function, if there exist $\underline{\alpha}, \rho \in \mathcal{K}_{\infty}$ with $\rho<$ id and $\gamma_{u} \in \mathcal{K}$ such that for all $x \in \mathbb{R}^{n}$, $\hat{x} \in \mathbb{R}^{\hat{n}}, \mathbf{x}_{[-h ; 0]} \in\left(\mathbb{R}^{n}\right)^{h+1}, \hat{\mathbf{x}}_{[-h ; 0]} \in\left(\mathbb{R}^{\hat{n}}\right)^{h+1}$ and $\hat{\mathrm{u}}_{[-h ; 0]} \in\left(\mathbb{R}^{\hat{m}}\right)^{h+1}$ there exists $\mathrm{u}_{[-h ; 0]} \in\left(\mathbb{R}^{m}\right)^{h+1}$ so that the following conditions hold

$$
\begin{aligned}
& \underline{\alpha}(|\hat{g}(\hat{x})-g(x)|) \leq V(\hat{x}, x), \\
& V\left(\hat{f}\left(\hat{\mathbf{x}}_{[-h ; 0]}, \hat{\mathrm{u}}_{[-h ; 0]}\right), f\left(\mathrm{x}_{[-h ; 0]}, \mathrm{u}_{[-h ; 0]}\right)\right) \\
& \leq \max \left\{\max _{i \in[-h ; 0]} \rho(V(\mathbf{x}(i), \hat{\mathbf{x}}(i))), \gamma_{u}\left(\left|\hat{\mathbf{u}}_{[-h ; 0]}\right|\right)\right\} .
\end{aligned}
$$

Now we establish that the existence of a Razumikhin simulation function ensures that output trajectories of the concrete and abtract systems lie within a certain vicinity of each other.

Theorem III.2 Consider systems $\Sigma^{d}$ and $\hat{\Sigma}^{d}$ with same output spaces. Let $V$ be a Razumikhin simulation function from $\hat{\Sigma}^{d}$ to $\Sigma^{d}$. Then there exist $\beta \in \mathcal{K} \mathcal{L}$ and $\gamma_{u} \in \mathcal{K}_{\infty}$ such that for all $\mathrm{x}_{[-h ; 0]} \in\left(\mathbb{R}^{n}\right)^{h+1}, \hat{\mathrm{x}}_{[-h ; 0]} \in\left(\mathbb{R}^{\hat{n}}\right)^{h+1}$ and $\hat{\overline{\mathbf{u}}} \in \hat{\overline{\mathcal{U}}}$ there exists $\overline{\mathbf{u}} \in \overline{\mathcal{U}}$ so that the following holds

$$
\begin{aligned}
& \left|\hat{\mathbf{y}}\left(k, \hat{\mathbf{x}}_{[-h ; 0]}, \hat{\overline{\mathbf{u}}}\right)-\mathbf{y}\left(k, \mathbf{x}_{[-h ; 0]}, \overline{\mathbf{u}}\right)\right| \\
& \quad \leq \max \left\{\beta\left(\max _{i \in[-h ; 0]} V(\mathbf{x}(i), \hat{\mathbf{x}}(i)), k\right), \gamma_{u}\left(|\hat{\mathbf{u}}|_{\infty}\right)\right\} .
\end{aligned}
$$

\section{A. Abstraction for linear systems}

Here we systematically construct a continuous abstraction for linear systems using a Razumikhin simulation function. In particular, we provide conditions in form of linear matrix inequities (LMIs) which can be solved efficiently.

We consider the following linear time-delay control systems as, respectively, a concrete and an abstract system.

$$
\Sigma^{l}:\left\{\begin{aligned}
\mathbf{x}(k+1) & =\sum_{i=0}^{h} A_{i} \mathbf{x}(k-i)+\sum_{i=0}^{h} B_{i} \mathbf{u}(k-i), \\
\mathbf{y}(k) & =C \mathbf{x}(k),
\end{aligned}\right.
$$

Assume that there exist a positive definite matrix $M$ such that the matrix inequality

$$
C^{\top} C \preceq M
$$

holds. Take the following Razumikhin simulation function candidate from $\hat{\Sigma}^{l}$ to $\Sigma^{l}$

$$
V(x, \hat{x})=(x-P \hat{x})^{\top} M(x-P \hat{x}),
$$

and let $\mathbf{u}(\cdot)$ be given by interface function $\nu$ as follows

$$
\begin{aligned}
\mathbf{u}(k-i) & =\nu(\mathbf{x}(k-i), \hat{\mathbf{x}}(k-i), \hat{\mathbf{u}}(k-i)) \\
& =R \hat{\mathbf{u}}(k-i)+Q \hat{\mathbf{x}}(k-i)+K(\mathbf{x}(k-i)-P \hat{\mathbf{x}}(k-i)),
\end{aligned}
$$

where $i \in[0 ; h]$ and $K, P, Q$ and $R$ are matrices of appropriate dimensions. Additionally, assume that the following equalities hold

$$
\begin{aligned}
& A_{i} P=P \hat{A}_{i}-B_{i} Q, \quad i \in[0 ; h], \\
& C P=\hat{C} .
\end{aligned}
$$

Let $\tilde{A}_{i}:=A_{i}+B_{i} K$ for $i \in[0 ; h]$. Assume that there exist $\varepsilon \in(0,1)$ such that

$$
\left[\begin{array}{ccc}
\tilde{A}_{0}^{\top} M \tilde{A}_{0} & \ldots & \tilde{A}_{0}^{\top} M \tilde{A}_{h} \\
\tilde{A}_{1}^{\top} M \tilde{A}_{0} & \ldots & \tilde{A}_{1}^{\top} M \tilde{A}_{h} \\
\vdots & \ddots & \vdots \\
\tilde{A}_{h}^{\top} M \tilde{A}_{0} & \ldots & \tilde{A}_{h}^{\top} M \tilde{A}_{h}
\end{array}\right]-\varepsilon\left[\begin{array}{cccc}
M & 0 & \ldots & 0 \\
0 & M & \ldots & 0 \\
\vdots & \vdots & \ddots & \vdots \\
0 & 0 & \ldots & M
\end{array}\right] \prec 0 .
$$

The next result shows that under the above conditions $V$ in (9) is a Razumikhin simulation function from $\hat{\Sigma}^{l}$ to $\Sigma^{l}$.

Corollary III.3 Consider systems $\Sigma^{l}$ and $\hat{\Sigma}^{l}$. Suppose that there exist matrices $M, P, Q$ and a scalar $\varepsilon \in(0,1)$ satisfying (8), 11, (12) and (13). Then $V$ defined by (9) is a Razumikhin simulation function from $\hat{\Sigma}^{l}$ to $\Sigma^{l}$ with the input function given by (10).

Necessary and sufficient conditions ensuring the existence of $P, Q, \hat{C}$ and $\hat{A}_{i}$ 's satisfying (11) and (12) can be concluded from [7, Lemma 2]. 


\section{INTERCONNECTED Systems With DElays}

In this section, compositional construction of interconnected systems with delay is considered. Typically there are two types of delay in interconnected systems: interconnection delays and local delays. Interconnection delays are due to receiving information from the neighboring subsystems with delay, whereas local delays arise in the dynamics of the individual subsystem. First, interconnection delays are only considered. We show that interconnection delays do not affect the compositional construction of abstractions for interconnected systems if standard small-gain arguments still hold. Then, local delays are taken into account. By integrating Razumikhin simulation functions with small-gain type arguments, compositional construction of abstractions for interconnected systems with local delay is established. Eventually, we merge both results to address a general scenario in which interconnected systems with both interconnection and local delays is considered.

\section{A. Interconnected systems with interconnection delay}

Consider the following subsystems

$$
\Sigma_{i}:\left\{\begin{aligned}
\mathbf{x}_{i}(k+1) & =f_{i}\left(\mathbf{x}_{i}(k), \mathbf{w}_{i[k-h ; k]}, \mathbf{u}_{i}(k)\right) \\
\mathbf{y}_{i}(k) & =g_{i}\left(\mathbf{x}_{i}(k)\right),
\end{aligned}\right.
$$

$i \in[1 ; \ell]$, with $\mathbf{x}_{i}(k) \in \mathbb{R}^{n_{i}}, \mathbf{u}_{i}(k) \in \mathbb{R}^{m_{i}}$, and the internal inputs $\mathbf{w}_{i[k-h ; k]}=\left[\mathbf{w}_{i}(k-h) ; \ldots ; \mathbf{w}_{i}(k)\right] \in \mathbb{R}^{h p_{i}}$ are partitioned as $\mathbf{w}_{i}(k-j)=\left[\mathbf{w}_{i 1}(k-j) ; \ldots ; \mathbf{w}_{i(i-1)}(k-\right.$ $\left.j) ; \mathbf{w}_{i(i+1)}(k-j) ; \ldots ; \mathbf{w}_{i \ell}(k-j)\right] \in \mathbb{R}^{p_{i}}$, for all $j \in[0 ; h]$, and partitioned outputs $\mathbf{y}_{i}(k)=\left[\mathbf{y}_{i 1}(k) ; \ldots ; \mathbf{y}_{i \ell}(k)\right] \in \mathbb{R}^{q_{i}}$, with $\mathbf{w}_{i j}(k) \in \mathbb{R}^{p_{i j}}, \mathbf{y}_{i j}(k) \in \mathbb{R}^{q_{i j}}$, and output function $g_{i}\left(\mathbf{x}_{i}(k)\right):=\left[g_{i 1}\left(\mathbf{x}_{i}(k)\right) ; \ldots ; g_{i \ell}\left(\mathbf{x}_{i}(k)\right)\right]$. Without loss of generality, we assume that all subsystems (14) share the same maximal delay $h$.

We interpret the outputs $\mathbf{y}_{i i}$ as external outputs, whereas the outputs $\mathbf{y}_{i j}$ with $i \neq j$ are internal outputs which are used to define the interconnected systems. In particular, we assume that $\mathbf{w}_{i j}=\mathbf{y}_{j i}$ for all $i, j \in[1 ; \ell], i \neq j$. Note that $g_{i j} \equiv 0$ if there is no connection from the $i$ th subsystem to the $j$ th subsystem. Given $\mathbf{w}_{i j}=\mathbf{y}_{j i}$ and aggrigating all the subsystems, one can have the overall network denoted by $\Sigma$.

Assume that there exist corresponding abstractions of each subsystem (14) with the following dynamics

$$
\hat{\Sigma}_{i}:\left\{\begin{aligned}
\hat{\mathbf{x}}_{i}(k+1) & =\hat{f}_{i}\left(\hat{\mathbf{x}}_{i}(k), \hat{\mathrm{w}}_{[k-h ; k]}, \hat{\mathbf{u}}_{i}(k)\right) \\
\hat{\mathbf{y}}_{i}(k) & =\hat{g}_{i}\left(\hat{\mathbf{x}}_{i}(k)\right),
\end{aligned}\right.
$$

with appropriate dimensions and the similar structure as those in (14). The resulting overall system obtained from interconnection of $\hat{\Sigma}_{i}$ 's is denoted by $\hat{\Sigma}$.

Here we aim to compositionally construct a simulation function from those of individual subsystems. To do this, we follow a similar strategy as that in Section III. More precisely, we introduce additional state variables by which we transform system $\Sigma$ (resp. $\hat{\Sigma}$ ) into a new interconnected system without delay, but of higher dimension. Then we associate with each subsystem of the new interconnected a local simulation function. Finally we apply a standard smallgain argument to construct an overall simulation function form individual simulation functions.

Starting with the first subsystem, i.e. $\Sigma_{1}$, in (14), we define $\mathbf{x}_{\ell+(j-1) h+i}(k):=\mathbf{w}_{i j}(k-(h-i+1)), i \in[1 ; h], 2 \leq j \leq \ell$. In that way, we introduce $(\ell-1) h$ additional state variables. Following this procedure for each subsystem $l \in[2 ; \ell]$, we have $\mathbf{x}_{\ell+(l-1)(\ell-1) h+(j-1) h+i}(k):=\mathbf{w}_{l j}(k-(h-i+1))$, $i \in[1 ; h], 1 \leq j \leq \ell, j \neq l$. The same applies to subsystems $\hat{\Sigma}_{i}$ 's. With these new variables one can transform the interconnected system $\Sigma$ (resp. $\hat{\Sigma}$ ) into a system without delay, which refer to as $\Sigma^{t}$ (resp. $\hat{\Sigma}^{t}$ ). Accordingly the subsystems of $\Sigma^{t}$ (resp. $\hat{\Sigma}^{t}$ ) are denoted by $\Sigma_{i}^{t}$ (resp. $\hat{\Sigma}_{i}^{t}$ ). Having defined the systems $\Sigma^{t}$ and $\hat{\Sigma}^{t}$, we make the following assumption on individual simulation functions for the first $\ell$ subsystems.

Assumption IV.1 Consider $\Sigma_{i}^{t}$, and $\hat{\Sigma}_{i}^{t} i \in[1 ; \ell]$. There exist functions $V_{i}: \mathbb{R}^{n_{i}} \times \mathbb{R}^{\hat{n}_{i}} \rightarrow \mathbb{R}_{\geq 0}, \underline{\alpha}_{i} \in \mathcal{K}_{\infty}, \gamma_{i j} \in \mathcal{K}_{\infty} \cup\{0\}$ and $\gamma_{i u} \in \mathcal{K}$ such that for all $k \in \mathbb{N}_{0}$, all $\mathbf{x}_{i}(k) \in \mathbb{R}^{n_{i}}$, all $\hat{\mathbf{x}}_{i}(k) \in \mathbb{R}^{\hat{n}_{i}}$, and all $\hat{\mathbf{u}}(k) \in \mathbb{R}^{\hat{m}_{i}}$ there exists $\mathbf{u}(k) \in \mathbb{R}^{m_{i}}$ so that we have

$$
\begin{aligned}
& \underline{\alpha}_{i}\left(\left|\hat{g}_{i}\left(\hat{\mathbf{x}}_{i}(k)\right)-g\left(\mathbf{x}_{i}(k)\right)\right|\right) \leq V_{i}\left(\hat{\mathbf{x}}_{i}(k), \mathbf{x}_{i}(k)\right), \\
& V_{i}\left(\hat{\mathbf{x}}_{i}(k+1), \mathbf{x}_{i}(k+1)\right) \leq \\
& \quad \max \left\{\max _{1 \leq j \leq \ell(\ell-1) h} \gamma_{i j}\left(V_{j}\left(\hat{\mathbf{x}}_{j}(k), \mathbf{x}_{j}(k)\right)\right), \gamma_{i u}(|\hat{\mathbf{u}}(k)|)\right\}
\end{aligned}
$$

where $V_{i}\left(\hat{\mathbf{x}}_{i}(k), \mathbf{x}_{i}(k)\right)=\left|\hat{\mathbf{x}}_{i}(k)-\mathbf{x}_{i}(k)\right|$ for all $\ell+1 \leq$ $i \leq \ell(\ell-1) h$.

Assumption IV.1 only makes dissipative conditions for the first $\ell$ subsystems. The lemma below provides an observation on dissipative conditions for the remaining subsystems. The proof follows from the relation between the additional state variables $x_{j}, \ell+1 \leq j \leq \ell(\ell-1) h$ with the internal inputs $w_{i j}$ and inequality 16 .

Lemma IV.2 Let Assumption IV.1 hold. Then the s-th subsystem for each $s=\ell+(l-1)(\ell-1) h+(j-1) h+i$, $i \in[1 ; h], 1 \leq j \leq \ell, j \neq l$, we have

$$
V_{s}\left(\hat{\mathbf{x}}_{s}(k+1), \mathbf{x}_{s}(k+1)\right) \leq \gamma_{s j}\left(V_{j}\left(\hat{\mathbf{x}}_{j}(k), \mathbf{x}_{j}(k)\right)\right.
$$

where $\gamma_{s j}:=\underline{\alpha}_{j}^{-1}$.

Now by direct application of classic small-gain arguments, e.g. [14, Theorem 7], one can conclude the following.

Proposition IV.3 Let Assumption IV.1 hold. Also suppose that the following holds

$$
\gamma_{i_{1} i_{2}} \circ \gamma_{i_{2} i_{3}} \circ \cdots \circ \gamma_{i_{r-1} i_{r}} \circ \gamma_{i_{r} i_{1}}<\mathrm{id}
$$

for all sequences $\left(i_{1}, \ldots, i_{r}\right) \in\{1, \ldots, \ell(\ell-1) h\}^{r}, r \in$ $[1 ; \ell(\ell-1) h]$, with gain functions $\gamma_{i j}$ with $1 \leq i \leq \ell$ satisfying (17) and those with $\ell+1 \leq i \leq \ell(\ell-1) h$ fulfilling 18. Then there exists a simulation function from $\hat{\Sigma}_{t}$ to $\Sigma_{t}$. 
As seen from Proposition IV.3, if small-gain condition (19) holds, construction of continuous abstractions for the interconnected system $\Sigma$ containing interconnection delay reduces to construction of continuous abstractions for the interconnected system $\Sigma^{t}$ containing no delay.

\section{B. Interconnected systems with local delay}

Consider an interconnection of $\ell \in \mathbb{N}, \ell \geq 2$ subsystems affected by local delays. Similar to $\Sigma^{d}$ in (5) but additionally with internal inputs and different output structure, each subsystem of the concrete network is given by

$$
\Sigma_{i}^{d}:\left\{\begin{aligned}
\mathbf{x}_{i}(k+1) & =f_{i}\left(\mathbf{x}_{i[k-h ; k]}, \mathbf{w}_{i}(k), \mathbf{u}_{i[k-h ; k]}\right) \\
\mathbf{y}_{i}(k) & =g_{i}\left(\mathbf{x}_{i}(k)\right),
\end{aligned}\right.
$$

where $\mathbf{w}_{i}(k)=\left[\mathbf{w}_{i 1}(k) ; \ldots ; \mathbf{w}_{i(i-1)}(k) ; \mathbf{w}_{i(i+1)}(k) ; \ldots\right.$, $\left.\mathbf{w}_{i \ell}(k)\right] \in \mathbb{R}^{p_{i}}, \mathbf{y}_{i}(k)=\left[\mathbf{y}_{i 1}(k) ; \ldots ; \mathbf{y}_{i \ell}(k)\right] \in \mathbb{R}^{q_{i}}$, with $i \in[1 ; \ell], \mathbf{w}_{i j}(k) \in \mathbb{R}^{p_{i j}}, \mathbf{y}_{i j}(k) \in \mathbb{R}^{q_{i j}}$, and output function $g_{i}\left(\mathbf{x}_{i}(k)\right):=\left[g_{i 1}\left(\mathbf{x}_{i}(k)\right) ; \ldots ; g_{i \ell}\left(\mathbf{x}_{i}(k)\right)\right]$. Note that $g_{i j} \equiv 0$ if there is no connection from subsystem $i$ to subsystem $j$. We assume that $\mathbf{w}_{i j}=\mathbf{y}_{j i}$ for all $i, j \in[1 ; \ell], i \neq j$. Subsystem $i$ of the abstract network, $\hat{\Sigma}_{i}^{d}$, is described by in a smilar way as for (20) with $\hat{\cdot}$ on the top. We make the following condition to provide a Razumikhin simulation function from local Razumikhin simulation functions.

Assumption IV.4 Suppose that for each $\Sigma_{i}^{d}$ and $\hat{\Sigma}_{i}^{d}$ with the same output spaces and for all $i \in[1 ; \ell]$, there exist a Razumikhin simulation function $V_{i}: \mathbb{R}^{n_{i}} \times \mathbb{R}^{\hat{n}_{i}} \rightarrow \mathbb{R}_{\geq 0}$ such that the following hold

(i) There exist functions $\underline{\alpha}_{i} \in \mathcal{K}_{\infty}$ such that for all $x_{i} \in$ $\mathbb{R}^{n_{i}}$ and all $\hat{x}_{i} \in \mathbb{R}^{\hat{n}_{i}}$ we have

$$
\underline{\alpha}_{i}\left(\left|\hat{g}_{i}\left(\hat{x}_{i}\right)-g_{i}\left(x_{i}\right)\right|\right) \leq V_{i}\left(\hat{x}_{i}, x_{i}\right) .
$$

(ii) There exist $\rho_{i \text { int }} \in \mathcal{K}_{\infty}, \gamma_{i i} \in \mathcal{K}_{\infty} \cup\{0\}$ and $\gamma_{i u} \in \mathcal{K}$ such that for all $\mathrm{x}_{i[-h ; 0]} \in\left(\mathbb{R}^{n}\right)^{h+1}$, $\hat{\mathrm{x}}_{i[-h ; 0]} \in\left(\mathbb{R}^{\hat{n}}\right)^{h+1}$, and $\hat{\mathrm{u}}_{i[-h ; 0]} \in\left(\mathbb{R}^{\hat{m}_{i}}\right)^{h+1}$ there exists $\mathrm{u}_{i[-h ; 0]} \in\left(\mathbb{R}^{m_{i}}\right)^{h+1}$ so that for all $\mathbf{w}_{i}(0) \in \mathbb{R}^{p_{i}}$, $\hat{\mathbf{w}}_{i}(0) \in \mathbb{R}^{\hat{p}_{i}}$ the following condition holds

$$
\begin{aligned}
& V_{i}\left(f_{i}\left(\mathbf{x}_{i[-h ; 0]}, \mathbf{w}_{i}(0), \mathbf{u}_{i[-h ; 0]}\right), \hat{f}_{i}\left(\hat{\mathbf{x}}_{i[-h ; 0]}, \hat{\mathbf{w}}_{i}(0), \hat{\mathbf{u}}_{i[-h ; 0]}\right)\right) \\
& \leq \max \left\{\max _{\theta \in[-h ; 0]} \gamma_{i i}\left(V_{i}\left(\mathbf{x}_{i}(\theta), \hat{\mathbf{x}}_{i}(\theta)\right)\right)\right. \\
& \left.\quad \rho_{i \operatorname{int}}\left(\left|\hat{\mathbf{w}}_{i}(0)-\mathbf{w}_{i}(0)\right|\right), \gamma_{i u}\left(\left|\hat{\mathbf{u}}_{i[-h ; 0]}\right|\right)\right\} .
\end{aligned}
$$

(iii) Let $\gamma_{i j}:=\rho_{i \mathrm{int}}(\ell-1) \underline{\alpha}_{j}^{-1}$ for $i \neq j$, where $\underline{\alpha}_{j} \in \mathcal{K}_{\infty}$ satisfying 21. Then the following condition holds

$$
\gamma_{i_{1} i_{2}} \circ \gamma_{i_{2} i_{3}} \circ \cdots \circ \gamma_{i_{r-1} i_{r}} \circ \gamma_{i_{r} i_{1}}<\mathrm{id}
$$

for all sequences $\left(i_{1}, \ldots, i_{r}\right) \in\{1, \ldots, \ell\}^{r}$ and $r \in$ $[1 ; \ell]$.

The following theorem presents an upper bound on the mismatch between the output trajectories of $\Sigma_{i}^{d}$ and $\hat{\Sigma}_{i}^{d}$.

Theorem IV.5 Let Assumption IV.4 hold. Then there exist $\beta \in \mathcal{K} \mathcal{L}, \gamma \in \mathcal{K}$ such that for all $\mathbf{x}_{[-h ; 0]} \in\left(\mathbb{R}^{n}\right)^{h+1}$, $\hat{\mathrm{x}}_{[-h ; 0]} \in\left(\mathbb{R}^{\hat{n}}\right)^{h+1}$ and $\hat{\overline{\mathbf{u}}} \in \hat{\overline{\mathcal{U}}}$, there exists $\mathbf{u} \in \overline{\mathcal{U}}$ so that the output trajectory of the abstract composite system of subsystems $\hat{\Sigma}_{i}^{d}$ and the concrete composite system of subsystems $\Sigma_{i}^{d}$ satisfy

$$
\begin{aligned}
& \left|\hat{\mathbf{y}}\left(k, \hat{\mathbf{x}}_{[-h ; 0]}, \hat{\overline{\mathbf{u}}}\right)-\mathbf{y}\left(k, \mathbf{x}_{[-h ; 0]}, \overline{\mathbf{u}}\right)\right| \leq \\
& \quad \max \left\{\beta\left(\max _{i \in[-h ; 0]} V(\mathbf{x}(i), \hat{\mathbf{x}}(i)), k\right), \gamma_{u}\left(|\hat{\overline{\mathbf{u}}}|_{\infty}\right)\right\} .
\end{aligned}
$$

Hence, it has been established that if every concrete subsystem and the associated abstract subsystem admit a local Razumikhin simulation function and the small-gain condition 23 holds, then the aggregation of $\hat{\Sigma}_{i}^{d}$ 's is an abstraction for the overall network of concrete subsystems.

\section{Interconnected systems with interconnection and local} delay

Consider a system with both interconnection and local delays. To deal with such a system, we combine the results from Sections IV-A and IV-B

Assume that the $i$ th concrete subsystem, $i \in[1 ; \ell]$, is described by

$$
\Sigma_{i}^{d}:\left\{\begin{aligned}
\mathbf{x}_{i}(k+1) & =f_{i}\left(\mathbf{x}_{i[k-h ; k]}, \mathrm{w}_{i[k-h ; k]}, \mathbf{u}_{i[k-h ; k]}\right), \\
\mathbf{y}_{i}(k) & =g_{i}\left(\mathbf{x}_{i}(k)\right),
\end{aligned}\right.
$$

with $\mathbf{x}_{i}: \mathbb{N}_{0} \rightarrow \mathbb{R}^{n_{i}}, \mathbf{y}_{i}: \mathbb{N}_{0} \rightarrow \mathbb{R}^{q_{i}}, \mathbf{u}_{i}: \mathbb{N}_{0} \rightarrow \mathbb{R}^{m_{i}}$ and with partitioned inputs and outputs as defined in (14). The $i$ th abstract subsystem, $i \in[1 ; \ell]$ is also described by

$$
\hat{\Sigma}_{i}^{d}:\left\{\begin{aligned}
\hat{\mathbf{x}}_{i}(k+1) & =\hat{f}_{i}\left(\hat{\mathbf{x}}_{i[k-h ; k]}, \hat{\mathbf{w}}_{i[k-h ; k]}, \hat{\mathbf{u}}_{i[k-h ; k]}\right), \\
\hat{\mathbf{y}}_{i}(k) & =\hat{g}_{i}\left(\hat{\mathbf{x}}_{i}(k)\right),
\end{aligned}\right.
$$

with appropriate dimensions and the similar structure as those in 25). In this case, we follow the same steps as those in Section IV-A to transfer the system into a system with a higher order but without interconnection delay. In that way, the resulting transferred system is in the form of system $\Sigma^{t}$ (resp. $\hat{\Sigma}^{t}$ ) in Section IV-A. Now by Theorem IV.5 if there exists a Razumikhin simulation function for the latter case, then one can show that the output trajectories of the composite system of subsystems 25 and that of subsystems 26 remain close to each other. This gives a general framework for interconnected systems with both interconnection and local time delays.

Remark IV.6 For linear systems, each subsystem 25 can be of the form

$$
\Sigma^{l}:\left\{\begin{aligned}
\mathbf{x}(k+1)= & \sum_{i=0}^{h}\left(A_{i} \mathbf{x}(k-i)+D_{i} \mathbf{w}(k-i)\right. \\
& \left.+B_{i} \mathbf{u}(k-i)\right), \\
\mathbf{y}(k)= & C \mathbf{x}(k) .
\end{aligned}\right.
$$

The abstract system can be also given similarly. Following lines in Section III-A we can provide an algorithmic procedure for computation of matrices $\hat{A}_{i}, \hat{B}_{i}$ and $\hat{C}$. Moreover, the input $\mathbf{u}(\cdot)$ be given by interface function $\nu$ as follows.

$$
\begin{aligned}
\mathbf{u}(k-i)= & \nu(\mathbf{x}(k-i), \hat{\mathbf{x}}(k-i), \hat{\mathbf{w}}(k-i), \hat{\mathbf{u}}(k-i)) \\
= & R \hat{\mathbf{u}}(k-i)+Q \hat{\mathbf{x}}(k-i)+K(\mathbf{x}(k-i)-P \hat{\mathbf{x}}(k-i)) \\
& +S \hat{\mathbf{w}}(k-i),
\end{aligned}
$$

where $i \in[0 ; h]$ and $K, P, Q, R$ and $S$ are matrices of appropriate dimensions. Compared with (10), here we additionally have $S \hat{\mathbf{w}}$. Finally $\hat{D}_{i}$ is computed by $D_{i}=P_{i} \hat{D}_{i}-B_{i} S_{i}$. 


\section{ILLUSTRATIVE EXAMPLE}

Here we verify our results via a numerical example. Consider a feedback interconnection of two subsystems, where each subsystem receives the information from the other one with a unit delay. Each subsystem $i=1,2$, of the from of 25], is described by

$\Sigma_{i}:\left\{\begin{array}{c}\mathbf{x}_{i}(k+1)=\sum_{j=0}^{1}\left(A_{i, j} \mathbf{x}_{i}(k-j)+D_{i, j} \mathbf{w}_{i}(k-j)\right. \\ \left.+B_{i, j} \mathbf{u}_{i}(k-j)\right), \\ \mathbf{y}_{i}(k)=C_{i} \mathbf{x}_{i}(k),\end{array}\right.$

where the system matrices are given by

$$
\begin{array}{rlrl}
A_{1,0} & =\left[\begin{array}{ccc}
1.01 & 0.3 & 0 \\
0 & 0.03 & 0.04 \\
0.03 & 0 & 0.08
\end{array}\right], & A_{1,1}=\left[\begin{array}{ccc}
0.03 & 0 & 0.05 \\
0.02 & 0.05 & 0.08 \\
0.092 & 0 & 0.2
\end{array}\right], \\
D_{1,0}^{\top}=\left[\begin{array}{lll}
0 & 0 & 0
\end{array}\right], & & D_{1,1}^{\top}=\left[\begin{array}{lll}
0.04 & 0.08 & 0.12
\end{array}\right], \\
B_{1,0}^{\top}=\left[\begin{array}{lll}
1 & 0 & 0
\end{array}\right], & B_{1,1}^{\top}=\left[\begin{array}{lll}
0 & 0 & 0.1
\end{array}\right], \\
C_{1}=\left[\begin{array}{lll}
1 & 0 & 0
\end{array}\right] . & &
\end{array}
$$

and

$$
\begin{array}{lll}
A_{2,0} & =\left[\begin{array}{ccc}
0.97 & 0.26 & 0.02 \\
0.02 & 0.1 & 0.02 \\
0 & 0 & 0.14
\end{array}\right], & A_{2,1}=\left[\begin{array}{ccc}
0.17 & 0.04 & 0.02 \\
0.08 & 0.12 & 0.1 \\
0.23 & 0.161 & 0.22
\end{array}\right], \\
D_{2,0}^{\top}=\left[\begin{array}{lll}
0 & 0 & 0
\end{array}\right], & D_{2,1}^{\top}=\left[\begin{array}{lll}
0.05 & 0.1 & 0.15
\end{array}\right], \\
B_{2,0}^{\top}=\left[\begin{array}{lll}
1 & 0 & 0
\end{array}\right], & B_{2,1}^{\top}=\left[\begin{array}{lll}
0 & 0 & 0.2
\end{array}\right], \\
C_{2}=\left[\begin{array}{lll}
1 & 0 & 0
\end{array}\right] . &
\end{array}
$$

Note that $D_{i, 0}^{\top}=\left[\begin{array}{lll}0 & 0 & 0\end{array}\right]$ in $(29)$ reflects that the fact that the information from the neighbor is not received immediately, but with a unit delay. From Remark IV.6, we use the following steps to construct an abstraction $\Sigma_{i}$ of each subsystem $\Sigma_{i}$. For each $i, j=1,2$, we do the following steps.

- Compute $M_{i}$ and $K_{i}$ so that $C_{i}^{\top} C_{i} \preceq M_{i}$ and inequality 13 holds.

- Determine $P_{i}$ and calculate $\hat{A}_{i, j}$ and $Q_{i}$ satisfying(11).

- Calculate $\hat{D}_{i, j}$ and $S_{i}$ satisfying the following equation

$$
D_{i, j}=P_{i} \hat{D}_{i, j}-B_{i, j} S_{i}
$$

- Obtain $\hat{C}_{i}$ satisfying $\hat{C}_{i}=C_{i} P_{i}$.

- Choose $\hat{B}_{i, j}$ and $R_{i}$ arbitrarily.

We start by computing $M_{1}, M_{2}, K_{1}$ and $K_{2}$ such that the matrix inequalities (8) and (13) holds. Taking $\varepsilon_{1}=\varepsilon_{2}=0.95$ in (13), we obtain

$M_{1}=\left[\begin{array}{ccc}987.48 & 19.64 & 1.1 \\ 19.64 & 985.28 & 0.65 \\ 1.1 & 0.65 & 970.78\end{array}\right], M_{2}=\left[\begin{array}{ccc}572.16 & 7.59 & 1.05 \\ 7.59 & 573.91 & 1.42 \\ 1.05 & 1.42 & 570.27\end{array}\right]$,

and $K_{1}=K_{2}=\left[\begin{array}{lll}-0.8 & -0.11 & 0\end{array}\right]$. In the next step, we determine $P_{1}$ and $P_{2}$ by $P_{1}^{\top}=P_{2}^{\top}=[1 ; 2 ; 3]$. Let $Q_{1}=-1.52$ and $Q_{2}=-1.41$. We obtain the subsystem $\hat{\Sigma}_{1}$ as $\hat{A}_{1,0}=-0.09, \hat{A}_{1,1}=0.18, \hat{D}_{1,0}=0$,

$\hat{D}_{1,1}=0.04, \quad \hat{B}_{1,0}=1, \quad \hat{B}_{1,1}=1, \hat{C}_{1}=1$, and the subsystem $\hat{\Sigma}_{2}$ as $\hat{A}_{2,0}=0.14, \hat{A}_{2,1}=0.31, \hat{D}_{2,0}=0$, $\hat{D}_{2,1}=0.05, \quad \hat{B}_{2,0}=1, \quad \hat{B}_{2,1}=1, \hat{C}_{2}=1$. The Razumikhin simulation function $V_{i}$ is given by

$V_{i}\left(\hat{\mathbf{x}}_{i}(k), \mathbf{x}_{i}(k)\right)=\left(\mathbf{x}_{i}(k)-P_{i} \hat{\mathbf{x}}_{i}(k)\right)^{\top} M_{i}\left(\mathbf{x}_{i}(k)-P_{i} \hat{\mathbf{x}}_{i}(k)\right)$, and $\mathbf{u}_{i}(k)$ is described by $\mathbf{u}_{i}(k)=R_{i} \hat{\mathbf{x}}_{i}(k)+Q_{i} \hat{\mathbf{x}}_{i}(k)+$ $K_{i}\left(\mathbf{x}_{i}(k)-P_{i} \hat{\mathbf{x}}_{i}(k)\right)+S_{i} \hat{\mathbf{w}}_{i}(k)$, where $R_{i}=1, Q_{1}=-1.52$, $Q_{2}=-1.41$ and $S_{1}=S_{2}=0$.

Following the procedure given in Section IV-A, we transform the overall system composed of subsystems (29) to a new interconnected system without interconnection delay. The same expression can be provided for the abstract system. Having transformed both systems into networks without interconnection delay, now we use Theorem IV.5 to verify the existence of a simulation function from $\hat{\Sigma}^{t}$ to $\Sigma^{t}$. To do so, we need to assure that the small-gain condition 23 holds. One can compute the coupling gains $\gamma_{i j}$ as $\gamma_{1 j}=0.6573$ for all $j \in[1 ; 4], \gamma_{2 j}=0.7647$ for all $j \in[1,4], \gamma_{32}=\left|C_{2}\right|=1$, $\gamma_{41}=\left|C_{1}\right|=1$ and the rest are all zero. Clearly these values of $\gamma_{i j}$ 's satisfy small-gain condition (23).

\section{REFERENCES}

[1] E. Fridman, Introduction to time-delay systems: Analysis and control. Springer, 2014.

[2] M. S. Mahmoud, Robust control and filtering for time-delay systems. CRC Press, 2000.

[3] P. Tabuada, Verification and control of hybrid systems: a symbolic approach. Boston, MA: Springer, 2009.

[4] A. Girard and G. J. Pappas, "Approximation metrics for discrete and continuous systems," IEEE Trans. Autom. Control, vol. 52, pp. 782 798, May 2007.

[5] R. Milner, Communication and Concurrency. Prentice-Hall international series in computer science, Upper Saddle River, NJ, USA: Prentice Hall, 1989.

[6] R. Alur, T. A. Henzinger, O. Kupferman, and M. Y. Vardi, "Alternating refinement relations," in Concurrency Theory: 9th International Conference, (Nice), pp. 163-178, 1998.

[7] A. Girard and G. J. Pappas, "Hierarchical control system design using approximate simulation," Automatica, vol. 45, no. 2, pp. 566-571, 2009.

[8] G. Pola, P. Pepe, and M. D. Di Benedetto, "Symbolic models for timevarying time-delay systems via alternating approximate bisimulation," Int. J. Robust Nonlinear Control, vol. 25, no. 14, pp. 2328-2347, 2015

[9] A. Girard, "Approximately bisimilar abstractions of incrementally stable finite or infinite dimensional systems," in 53rd IEEE Conf. Decision Control, (Los Angeles), pp. 824-829, Dec 2014.

[10] G. Pola, P. Pepe, M. D. D. Benedetto, and P. Tabuada, "Symbolic models for nonlinear time-delay systems using approximate bisimulations," Syst. Control Lett., vol. 59, no. 6, pp. 365-373, 2010.

[11] E. Fridman, "Tutorial on Lyapunov-based methods for time-delay systems," Euro. J. Control, vol. 20, no. 6, pp. 271-283, 2014.

[12] R. H. Gielen, M. Lazar, and A. R. Teel, "Input-to-state stability analysis for interconnected difference equations with delay," Math Control Signals Syst., vol. 24, no. 1-2, pp. 33-54, 2012.

[13] M. Rungger and M. Zamani, "Compositional construction of approximate abstractions of interconnected control systems," IEEE Trans. Control Netw. Syst., vol. 5, no. 1, pp. 116-127, 2018.

[14] N. Noroozi, F. R. Wirth, and M. Zamani, "Compositional construction of abstractions via relaxed small-gain conditions Part I: continuous case," in Euro. Control Conf., (Limassol), pp. 76-81, 2018.

[15] S. N. Dashkovskiy, B. S. Rüffer, and F. R. Wirth, "Small gain theorems for large scale systems and construction of ISS Lyapunov functions," SIAM J. Control Optim., vol. 48, no. 6, pp. 4089-4118, 2010.

[16] H. K. Khalil, Nonlinear Systems. Englewood Cliffs, NJ: Prentice-Hall, 3rd ed., 2002.

[17] A. R. Teel, "Connections between Razumikhin-type theorems and the ISS nonlinear small gain theorem," IEEE Trans. Autom. Control, vol. 43, no. 7, pp. 960-964, 1998. 